\title{
Position and Force Teaching Method for 6 DoF Manipulator Using Contact Teaching Tool and Teaching Data Editor
}

\author{
Duy-Do Bui \\ Department of Electrical and Mechanical Engineering, Graduate School of Engineering, \\ Nagoya Institute of Technology, Gokiso-cho, Showa-ku, Nagoya, Aichi 466-8555, Japan \\ Hiroki Tanaka, Quang-Trung Chu, Hideki Inuzuka, Yoshifumi Morita \\ Department of Electrical and Mechanical Engineering, Graduate School of Engineering, \\ Nagoya Institute of Technology, Gokiso-cho, Showa-ku, Nagoya, Aichi 466-8555, Japan \\ Masao Sakai \\ Industrial Research Center, Aichi Center for Industry and Science Technology \\ Onda-cho 1-157-1, Kariya-shi Aichi 448-0013, Japan \\ E-mail: cmc13126@nitech.jp,h.tanaka.683@nitech.jp, \\ h.inuzuka.776@nitech.jp,morita@nitech.ac.jp,sakai@aichi-inst.jp \\ www.nitech.ac.jp
}

\begin{abstract}
This paper proposes a direct teaching method for teaching tasks performed by a robot manipulator while the robot's tool makes contact with a workpiece. A contact teaching tool designed for accurately grasping the shape of the workpiece, and a teaching data editor for generating the target trajectory to realize the desired press angle and force, are developed on the assumption that the compliance characteristics of the robot hand are known. The effectiveness of the developed method is confirmed experimentally.
\end{abstract}

Keywords: Direct teaching method, Robot manipulator, Contact teaching tool, Teaching data editor.

\section{Introduction}

In the manufacturing industry, efficient robot teaching technologies are essential to bring out the fullest potential of robot manipulators. Currently, for the teaching task of robots, the teaching pendant is widely employed. However, it is difficult to teach complex trajectories by the teaching pendant. Moreover, this method consumes a significant amount of time to teach robots. Therefore, new direct teaching methods have been investigated. ${ }^{1,2}$ To solve these problems, a parallel wire-type teaching device (PAWTED) was developed to directly teach robot manipulators using human hands instead of teaching pendants. ${ }^{3}$ Nonetheless, it is not possible to determine teaching points precisely by the PAWTED since the operator has to visually checks and adjusts by hand.

Therefore, in this study, a contact teaching tool and a teaching data editor were proposed on the assumption that the compliance characteristics of the robot hand are known. The tool allows us to accurately grasp the shape of the workpiece just by pressing the teaching tool against the edges of the workpiece. Subsequently, the editor will generate the target trajectory to realize the desired pressing angle and force based on the obtained teaching data and the robot hand characteristics. The effectiveness of the proposed method was confirmed experimentally from the viewpoint of improving the teaching accuracy and reducing the teaching time.

(C) The 2021 International Conference on Artificial Life and Robotics (ICAROB2021), January 21 to 24, 2021 


\section{PAWTED with a contact teaching tool}

The PAWTED consists of a moving platform, six wires, and a base. The base, which is attached to an industrial six-degree-of-freedom (6-DoF) manipulator, has six rotary encoders and six flat spiral springs. The moving platform is connected to the base by six metal wires. As a result, the moving platform can move freely in threedimensional space within the working area.

The operation of the PAWTED comprises teaching and playback modes. In the teaching mode, when the operator moves the moving platform, the system will compute the position of the end-effector based on the drawn length of the six wires. Then, the robot will be controlled to track and maintain a constant distance with the moving platform. In the playback mode, the moving platform is fixed to the base. Subsequently, the teaching trajectory is reproduced by the robot.

In this research, we aimed to develop a contact teaching tool that allows the teaching position to be obtained easily just by touching the contact teaching tool to the edge of the workpiece. Therefore, a contact teaching tool with a design as in Fig. 1 (a) is proposed. As shown in Fig. 1 (b), $p_{a}$ and $p_{b}$ are the two teaching points of the teaching tools. The midpoint of the line segment connecting $p_{a}$ and $p_{b}$, which is the rotation center point of these teaching points is denoted as $p_{0}$. To improve the flexibility of the teaching tool, it is designed so that it can rotate freely around its central axis as depicted in Fig. 1 (a). Besides, due to the empty v-shaped structure, the contact teaching tool could be used to make contact with both straight edge and arc-shaped edge types workpieces.

In the teaching mode, the contact teaching tool is attached to a moving platform, as illustrated in Fig. 2 . When the operator touches the contact teaching tool to the edge of the workpiece, $p_{a}$ and $p_{b}$ will make contact with the edge of the workpiece and the position and orientation of the teaching tool are determined. After teaching the trajectory, the editor calculates the trajectory of the teaching points, and desired trajectory on the playback mode. In this study, a robot hand with compliance characteristics and a robot's tool attached to the robot hand were simulated as a joystick in the playback mode. The compliance characteristics of the robot hand are considered to be the compliance characteristics of the joystick.

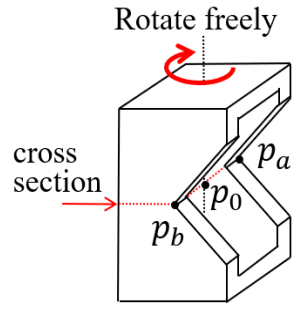

(a) Contact teaching tool
Rotation center point of teaching points

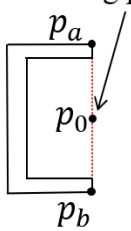

b) Tool's cross section
Fig. 1. Contact teaching tool structure

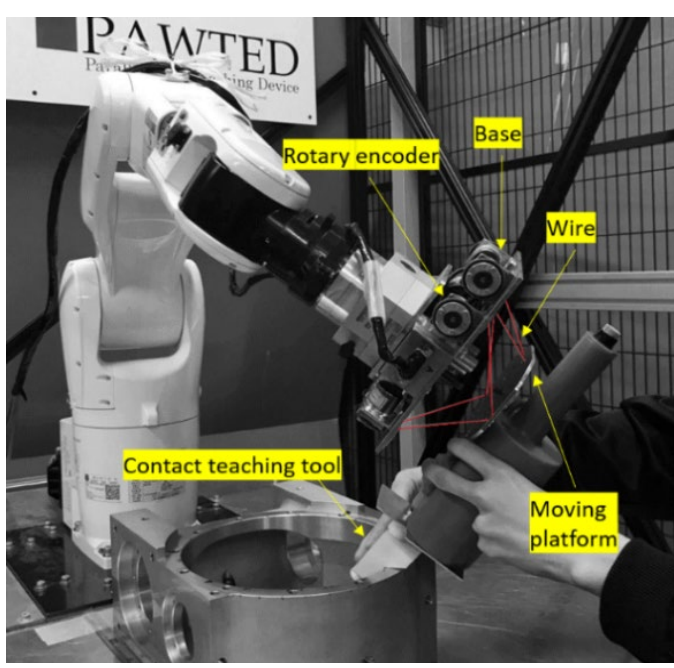

Fig. 2. PAWTED with a contact teaching tool

\section{Teaching data editor}

A teaching data editor generates the target trajectory to realize the desired pressing angle and force based on the teaching trajectory and the characteristics of the contact teaching tool and playback tool.

A model diagram of the computing process in order to obtain the circular arc-shaped trajectory for the robot is illustrated in Fig. 3. $p_{a i}$ and $p_{b i}$ are the teaching positions obtained when the contact teaching tool touches the edge of the workpiece $(i=1,2, \cdots, n)$. The rotation center point of $p_{a i}$ and $p_{b i}$ is denoted as $p_{0 i} . S$ is the auxiliary circular trajectory created by multiple $p_{0 i}$ points which can be calculated based on the PAWTED moving platform. $C_{0}$ is the center of $S$ and the hole of the workpiece. The radii of $S$ and the hole are $r_{1}$ and $r_{2}$, respectively. After at least three rotation center points $p_{0 i}$ are obtained, the least squares fitting method is applied to precisely determine $C_{0}$ and $r_{1}$ from multiple $p_{0 i}$ points. 


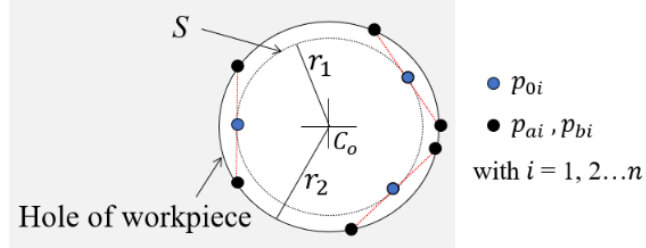

Fig. 3. Getting the hole trajectory
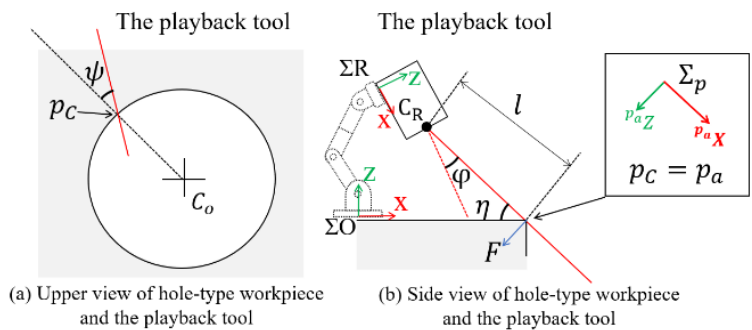

Fig. 4. Hole-type workpiece and playback tool.

Afterwards, the position of an arbitrary number of the teaching points $p_{a i}$ and $p_{b i}$ can be determined from $S$. Subsequently, the least squares fitting method is adopted once again to acquire the radius $r_{2}$ of the circular trajectory that we need to teach the robot. From the newly obtained teaching trajectory, the tool orientation at the time of teaching can be changed to the required orientation.

In Fig. $4, p_{C}$ is denoted as the contact position between the playback tool and the edge of the workpiece. $C_{R}$ is the center pivot point of the playback tool and $l$ is the distance between $C_{R}$ and $p_{C}$. The angle between the line that connect $p_{C}$ and $C_{0}$ and the playback tool is denoted as $\psi$, as shown in Fig. 4 (a). In Fig. 4 (b), $F$ is the force that the playback tool presses against the workpiece. $\eta$ is the angle between the workpiece surface and the playback tool when viewed from the side of the workpiece. The inclination angle between the symmetrical axis of the playback tool and its joystick when it is pressed against the workpiece is denoted as $\varphi$. The pressing force $F$ is adjusted based on $\varphi$. Based on the compliance characteristics between $\varphi$ and $F, \varphi$ can be approximated as a function of $F$ as follows:

$$
\varphi=f(F)
$$

In this study, for simplicity of calculation, we chose $p_{a}$ as our teaching point. In the coordinate frame $\Sigma_{P}$ which is attached to $p_{a}$, the position and orientation of the teaching point $p_{a}$ is calculated to be the desired contact position and orientation of the robot's tool. The position and orientation of $C_{R}$ as follows:

$$
{ }_{C_{R}}^{o} T={ }_{p_{C}}^{o} T\left[\begin{array}{cc}
{ }^{p_{C}} R & { }^{p_{C}} X \\
0 & 1
\end{array}\right]
$$

in which $C_{R}^{O} T$ is the simultaneous transformation matrix from frame $\Sigma_{O}$ to $\Sigma_{C_{R}} . \quad p_{C}^{O} T$ is the simultaneous transformation matrix from frame $\Sigma_{O}$ to $\Sigma_{p_{C}} \cdot{ }_{C_{R}}^{p_{C}} X$ denotes the translation of $-l$ in the $X$ axis of frame $\Sigma_{P}$ and ${ }_{C_{R}}^{p_{C}} R$ denotes the rotation of $-\varphi$ about the $\mathrm{Y}$ axis of frame $\Sigma_{P}$.

$$
\begin{gathered}
{ }_{C_{R}} R=R_{Y}(\varphi) \\
{ }_{C_{R}} X=\left[\begin{array}{lll}
-l & 0 & 0
\end{array}\right]^{T}
\end{gathered}
$$

Finally, since the simultaneous transformation matrix from the center of rotation frame $\Sigma_{C_{R}}$ to the robot end-effector frame $\Sigma_{R}$ is known as ${ }_{R}^{C_{R}} T$, the target position and orientation $\mathrm{R}$ of the robot at the teaching points is obtained as:

$$
{ }_{R}^{O} T={ }_{C_{R}}^{O} T_{R}^{C_{R}} T
$$

where ${ }_{R}^{O} T$ is the simultaneous transformation matrix from frame $\Sigma_{O}$ to $\Sigma_{R}$ whose position and orientation are target coordination of the robot end-effector to realize the desired trajectory.

\section{Experiment}

In order to verify the effectiveness of the new direct teaching method with the PAWTED, a contact teaching tool, and a data editor (PAWTED + ce), experiments were carried out on the 6-DoF industrial robot (DENSO WAVE, VS-060). Specifically, we have performed the experiments with the task of teaching a circular arcshaped trajectory for the robot, as shown in Fig. 5 (a). The experimental results are compared with the other two methods that used a teaching pendant and PAWTED. For evaluation purposes, each of the three teaching methods is performed three times, and their average value is used.

In the teaching mode, to create the same teaching condition, the operator taught the trajectory that makes the desired values of $l=45 \mathrm{~mm}, \psi=0^{\circ}, \eta=45^{\circ}$, and $F=125 \mathrm{gf}$ for the robot, as illustrated by the red lines in Fig. 6-9, respectively. Afterwards, in the playback mode, 


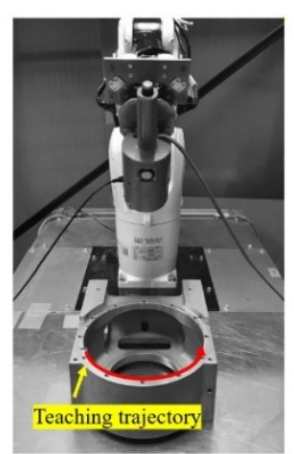

(a) Circular arc trajectory

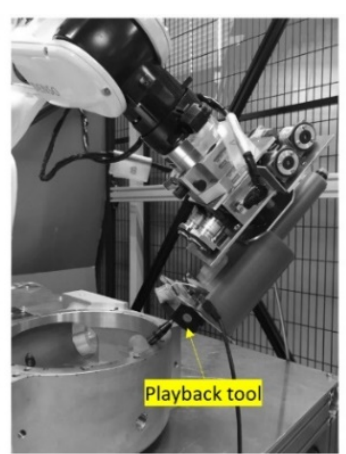

(b) Teaching conditions of the tool
Fig. 5. Teaching trajectory and conditions.

a joystick is used as a playback tool to perform the evaluation experiments, as illustrated in Fig. 5 (b). Based on the compliance characteristics of the joystick, the target position of the manipulator on the playback mode can be determined according to Eq. (5).

In this study, our objective is optimizing the playback trajectory so that the robot can make a contact with the workpiece precisely. The parameters $l, \psi, \eta$ and $F$ for the desired positions and orientations of the robot's tool are evaluated. The experiment results of $l, \psi, \eta$ and $F$, which are analyzed with the unequal variances t-test, are shown in Figs. 6-9, respectively. * shows statistically significant at $p<0.05$. Regarding the maximum average errors of each parameter, among the three teaching methods, the PAWTED + ce has standout performances.

The teaching-editing time of the three teaching methods for a circular arc trajectory with 11 teaching points is shown in Table 1. Since the position and orientation of the PAWTED + ce method are determined just by touching the teaching tool to the edge of the workpiece, it is possible to quickly teach any point on the edge of the workpiece for the robot. As shown in Table

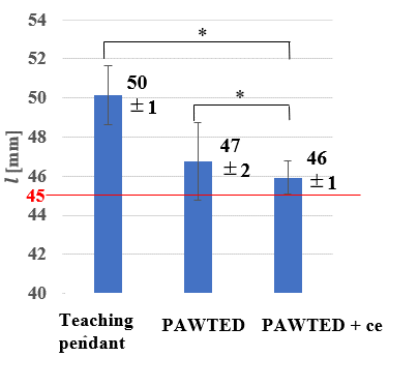

Fig. 6. Teaching distance $l$ on the teaching points.

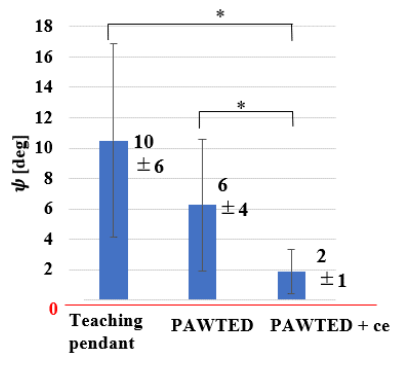

Fig. 7. Teaching angle $\psi$ on the teaching points.

1, the average teaching-editing time in case of using PAWTED + ce, PAWTED, and the teaching pendant are $11 \mathrm{sec}, 29 \mathrm{sec}$ and $104 \mathrm{sec}$, respectively. PAWTED + ce method reduced the teaching-editing time significantly.

Table 1. Teaching-editing time for a circular arc trajectory.

\begin{tabular}{lcc} 
& Average & Standard deviation \\
\hline Teaching pendant & $104 \mathrm{sec}$ & $46.0 \mathrm{sec}$ \\
PAWTED & $29 \mathrm{sec}$ & $13.0 \mathrm{sec}$ \\
PAWTED + ce & $11 \mathrm{sec}$ & $2.4 \mathrm{sec}$ \\
\hline
\end{tabular}

\section{Conclusion}

This study presented a new method for teaching tasks performed by a robot manipulator while the robot's tool makes contacts with the workpiece. We investigated the performance of the proposed teaching method in the task of teaching a circular arc-shaped trajectory for the industrial robot. From the experimental results compared with the conventional methods, it has been shown that the proposed teaching method has the highest teaching position accuracy and the shortest teaching time. A future plan is to verify the usefulness of the proposed method in the actual field. This research was supported by Aichi Prefectural Government through "Knowledge Hub Aichi," Priority Research Project (II) and in the experiments by Kondo Seisakusho Co., Ltd. and FINE TECHNO Co., Ltd.

\section{References}

1. S. Masaru, Direct Teaching for Robot, J. Robotics Society of Japan. 13(5), 1995, pp. 627-628.

2. Y. Mochizuki and T. Matsui, Direct Teaching Method for Hybrid Control Applying Impedance Control, J. Robotics Society of Japan. 12(2), 1994, pp. 160-166.

3. S. Sakai, S. Noritaka, M. Yoshifumi, Development of Parallel Wire Mechanism for Robot Teaching, J. Japan Society of Mechanical Engineers Series C. 79(807), 2013, pp. 4321-4329.

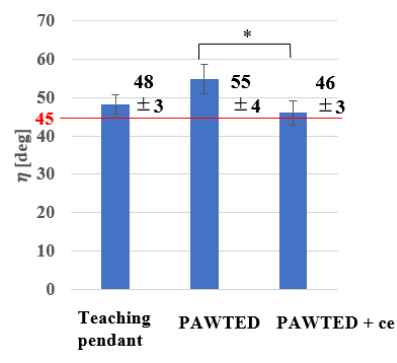

Fig. 8. Teaching angle $\eta$ on the teaching points.

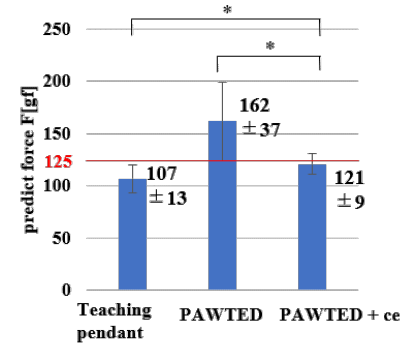

Fig. 9. Teaching force $F$ on the teaching points. 